\title{
Characterisation of transcription factors in rainbow trout potentially involved in dendritic cell maturation and activation
}

\author{
Jun Wang ${ }^{1,3}$, Tiehui Wang ${ }^{1}$, Ottavia Benedicenti ${ }^{1}$, Catherine Collins ${ }^{2}$, Una McCarthy \\ 2, Kaiyu Wang ${ }^{3}$, Christopher J Secombes ${ }^{1}$, Jun Zou ${ }^{1 \S}$ \\ ${ }^{1}$ Scottish Fish Immunology Research Centre, Institute of Biological and Environmental \\ Sciences, University of Aberdeen, Aberdeen, AB24 2TZ, UK \\ ${ }^{2}$ Marine Scotland Science Marine Laboratory, 375 Victoria Rd, Aberdeen AB11 9DB, UK \\ ${ }^{3}$ Department of Basic Veterinary, Sichuan Agricultural University, Huimin Road No. 211, \\ Chengdu 611130, China
}

\begin{abstract}
Dendritic cells (DCs) are heterogeneous cell populations derived from the bone marrow in mammals and play essential roles in antigen presentation and activation of innate and adaptive immune responses after infection. Phenotyping DCs mainly relies on expression of multiple cell surface markers, making it difficult for comparative studies in different species. Recently several transcription factors (TFs) controlling DC differentiation and maturation have been characterised in humans and mice and are highly conserved. These include zinc finger and BTB domain containing 46 (Zbtb46), zinc finger protein 366 (Znf366) and basic leucine zipper transcription factor/ATF-like 3 (Batf3). To search for common molecular markers to assist identification of DC like cells in fish, in this study the gene homologues for these DC-TFs were sequenced in rainbow trout and their phylogeny was analysed. Gene expression was studied in tissues of healthy fish and in gills of Atlantic salmon after exposure to Paramoeba perurans. Furthermore, monocytes/macrophages were isolated from rainbow trout and cultured in the presence of recombinant interleukin 4/13 for 7 days and then examined for DC-TF expression after stimulation with Toll-like receptor ligands such as lipopolysaccharide, bacterial flagellin, polyI:C and imidazoquinoline.
\end{abstract}

\section{Key words}

Fish dendritic cell transcription factor

Corresponding author. Tel.: +44 1224273796.

Email: j.j.zou@abdn.ac.uk. 